\title{
REVIEW ARTICLE The antibody/microbiota interface in health and disease
}

\author{
Delphine Sterlin ${ }^{1,2}$, Jehane Fadlallah ${ }^{3}$, Emma Slack $^{4}$ and Guy Gorochov ${ }^{1}$
}

The human intestine is densely colonized with commensal microbes that stimulate the immune system. While secretory Immunoglobulin (Ig) A is known to play a crucial role in gut microbiota compartmentalization, secretory lgM, and systemic lgG have recently been highlighted in host-microbiota interactions as well. In this review, we discuss important aspects of secretory lgA biology, but rather than focusing on mechanistic aspects of IgA impact on microbiota, we stress the current knowledge of systemic antibody responses to whole gut microbiota, in particular their generation, specificities, and function. We also provide a comprehensive picture of secretory lgM biology. Finally, therapeutic and diagnostic implications of these novel findings for the treatment of various diseases are outlined.

Mucosal Immunology (2020) 13:3-11; https://doi.org/10.1038/s41385-019-0192-y

\section{INTRODUCTION}

Humoral immunity, in particular antibody-mediated responses, plays a key role in the host defense against extracellular pathogens. Notably, the function of secretory Immunoglobulin (Ig) A, given its mucosal localization, has been considered for a long time to be restricted to immune exclusion, i.e., neutralization of luminal pathogens prevention of host invasion. In the past decade, with the advent of microbial metagenomics and the improvement of bacterial flow cytometry, murine and human studies have shed new light on $\mathrm{lgA} /$ microbiota commensalism. $\lg A$ is now viewed as playing a pivotal role in supporting the establishment, selection, diversification, and long-term maintenance of a healthy commensal gut microbiota.

Novel functions have also been ascribed, albeit putatively, to $\lg M$ and $\lg G$ at the gut-microbial interface. Whereas early studies have shown the presence of IgM plasmocytes in gut biopsies of $\operatorname{lgA}$-deficient patients $(\operatorname{lgAd}),{ }^{1,2}$ more recent studies provide evidence that secretory $\operatorname{lgM}(\mathrm{s} \lg M)$ binds a fraction of commensal microbiota in IgA deficiency thereby enhancing bacterial diversity. ${ }^{3}$ Moreover, slgM in healthy humans seem to bind a very restricted fraction of gut microbiota along with secretory $\lg A .{ }^{4}$ The functional consequences of these observations are unclear, but could be clinically relevant as it is well established that patients selectively lacking IgA rarely develop inflammatory bowel diseases (IBD), which is instead more common and severe in those lacking both $\lg A$ and $\operatorname{lgM}{ }^{5,6}$ Finally, systemic lgG responses targeting gutmicrobial communities were initially described in IBD and considered merely secondary to bacterial translocations in relation with gut barrier defects. However, it has been reported recently that IgG targeting either bacterial antigens, whole bacterial cells, or whole microbial consortia, can also be retrieved from healthy subjects.

Here, we review recent murine and human studies in an attempt to provide a comprehensive picture of our current knowledge of the interplay between gut antibodies and microbiota.

\section{SECRETORY IGA BIND A WIDE RANGE OF COMMENSALS}

Early studies of gut microbiota revealed that secretory IgA coat only a fraction of human and murine microbiota. ${ }^{7,8}$ Recently, a combination of bacterial flow cytometry with high-throughput $16 \mathrm{~S}$ gene sequencing offered an opportunity to identify $\lg \mathrm{A}$ coated bacteria ex vivo. Several such IgAseq studies have independently described $\lg \mathrm{A}^{+}$communities in mice ${ }^{9-14}$ and in humans., 3,15-18 Table 1 gathers bacterial taxa that are significantly targeted by $\lg \mathrm{A}$ in healthy individuals. Despite some variations, core features of the IgA-coated subset emerge from these data. First, IgA bound a highly diverse fraction of microbiota, including numerous rare genera (generally $<1 \%$ in frequency). Second, the four major phyla, namely Actinobacteria, Bacteroidetes, Firmicutes, and Proteobacteria were detected in IgA-coated microbiota. Consistently, most microbes, irrespective of their microbial phylogeny, can induce specific IgA in monocolonized gnotobiotic mice. ${ }^{19,20}$ Of note, multiple taxa of the phylum Firmicutes, in particular those belonging to the family Lachnospiraceae, are significantly enriched within the $\operatorname{lgA}^{+}$fraction whatever the human cohort (Table 1). The relatively high frequency of these organisms in the human colon might account for their prevalence in $\operatorname{lgA}^{+}$fractions. This observation supports earlier studies showing that Lachnospiraceae members are able to elicit innate immune responses and modulate adaptive immune responses in the gut. ${ }^{21-23}$

While it was concluded from each of the above mentioned studies that $\lg A$ preferentially binds a taxonomically distinct subset of the whole microbiota, it remains difficult to define a core slgA-coated community in healthy individuals. A similar observation has been made in uniform patient groups. One likely reason is that interstudy variability might not only result from technical factors, but also from different strategies to define the slgA-coated microbiota. Key variations in immunostaining protocols, such as the use of either monoclonal ${ }^{4,24}$ or polyclonal anti-lgA antibody, $3,9,15,16,18$ have a substantial impact on background staining.

\footnotetext{
${ }^{1}$ Sorbonne Université, Inserm, Centre d'Immunologie et des Maladies Infectieuses (CIMI-Paris), AP-HP Hôpital Pitié-Salpêtrière, 75013 Paris, France; ${ }^{2}$ Unit of Antibodies in Therapy

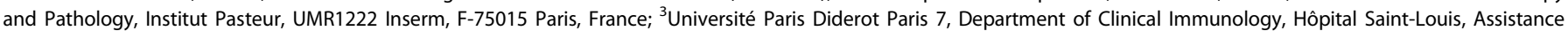
Publique Hôpitaux de Paris (APHP), EA3518, 75010 Paris, France and ${ }^{4}$ Institute of Food Sciences, Nutrition and Health, ETH Zurich, 8093 Zürich, Switzerland Correspondence: Emma Slack (emma.slack@hest.ethz.ch) or Guy Gorochov (guy.gorochov@sorbonne-universite.fr)
} 
Table 1. IgA-coated bacteria in humans

\begin{tabular}{|c|c|c|c|c|c|}
\hline Study & Cohort & Sequencing method & Family & Genera & Species \\
\hline \multirow{8}{*}{$\begin{array}{l}\text { D'Auria et al, 2013, Sci } \\
\text { Rep (18) }\end{array}$} & \multirow[t]{8}{*}{6 healthy adults } & \multirow[t]{8}{*}{ 16S gene sequencing } & Bacteroidaceae & ND & ND \\
\hline & & & Ruminococcaceae & & \\
\hline & & & Enterococcaceae & & \\
\hline & & & Lachnospiraceae & & \\
\hline & & & Nocardiaceae & & \\
\hline & & & Pseudomonaceae & & \\
\hline & & & Comamonadaceae & & \\
\hline & & & $\begin{array}{l}\text { Sphingomonadaceae } \\
\text { ud-Sphingomonadales }\end{array}$ & & \\
\hline \multirow{6}{*}{$\begin{array}{l}\text { Palm et al, 2014, Cell } \\
\text { (24) }\end{array}$} & \multirow{6}{*}{20 healthy adults } & \multirow{6}{*}{$16 \mathrm{~S}$ gene sequencing } & Actinomycetaceae & Actinomyces & spp. \\
\hline & & & Bifidobacteriaceae & Bifidobacterium & ND \\
\hline & & & Erysipelotrichaceae & Erysipelotrichaceae & ND \\
\hline & & & Lachnospiraceae & Dorea & spp. \\
\hline & & & Lachnospiraceae & Ruminococcus & torques \\
\hline & & & Verrucomicrobiaceae & Akkermansia & muciniphila \\
\hline \multirow{4}{*}{$\begin{array}{l}\text { Kau et al, 2015, Sci } \\
\text { Transl Med (15) }\end{array}$} & \multirow{4}{*}{11 healthy children } & \multirow{4}{*}{$16 \mathrm{~S}$ gene sequencing } & Clostridiaceae & ND & \\
\hline & & & Streptococcaceae & Streptococcus & salivarius \\
\hline & & & Enterobacteriaceae & Escherichia/Shigella & ND \\
\hline & & & Verrucomicrobiaceae & Akkermansia & muciniphila \\
\hline \multirow{6}{*}{$\begin{array}{l}\text { Planer et al, 2016, } \\
\text { Nature (16) }\end{array}$} & \multirow[t]{6}{*}{82 twins, 2 year-old } & \multirow[t]{6}{*}{165 gene sequencing } & Bifidobacteriaceae & Bifidobacterium & bifidum \\
\hline & & & Clostridiaceae & Clostridium & nexile \\
\hline & & & Clostridiaceae & Clostridium & spiroforme \\
\hline & & & Lachnospiraceae & Ruminococcus & torques \\
\hline & & & Lachnospiraceae & Ruminococcus & gnavus \\
\hline & & & Enterobacteriaceae & Escherichia/Shigella & ND \\
\hline \multirow{6}{*}{$\begin{array}{l}\text { Dzidic et al, 2016, J } \\
\text { Allergy Clin Immunol } \\
\text { (17) }\end{array}$} & \multirow{6}{*}{$\begin{array}{c}28 \text { healthy children } \\
1 \text { year-old }\end{array}$} & \multirow[t]{6}{*}{$16 \mathrm{~S}$ gene sequencing } & Bacteroideaceae & Bacteroides & ND \\
\hline & & & Clostridiaceae & ND & ND \\
\hline & & & Clostridiales Famille XI & ND & ND \\
\hline & & & Erysipelotrichaceae & ND & ND \\
\hline & & & Erysipelotrichaceae & ND & ND \\
\hline & & & Lachnospiraceae & Roseburia & ND \\
\hline \multirow{10}{*}{$\begin{array}{l}\text { Magri et al, 2017, } \\
\text { Immunity (4) }\end{array}$} & \multirow[t]{2}{*}{5 healthy adults } & \multirow[t]{10}{*}{$16 \mathrm{~S}$ gene sequencing } & Bacteroideaceae & Bacteroides & ND \\
\hline & & & Barnesiellaceae & ND & ND \\
\hline & \multirow{8}{*}{$\begin{array}{l}\text { Mucus-embedded } \\
\text { microbiota }\end{array}$} & & Erysipelotrichaceae & ND & ND \\
\hline & & & Lachnospiraceae & ND & ND \\
\hline & & & Lachnospiraceae & Roseburia & ND \\
\hline & & & Lachnospiraceae & Blautia & ND \\
\hline & & & Lachnospiraceae & Ruminococcus & ND \\
\hline & & & Lachnospiraceae & Ruminococcus & gnavus \\
\hline & & & Ruminococcaceae & & ND \\
\hline & & & Ruminococcaceae & Ruminococcus & ND \\
\hline
\end{tabular}

Taxa belonging to Actinobacteria are colored in pink, Bacteroidetes in green, Proteobacteria in blue, Verrucomicrobia in purple, and Firmicutes in orange

Differences in sequencing methods may also explain the variations observed between reports. $\lg \mathrm{A}^{+}$bacterial identification usually relies on $16 \mathrm{~S}$ gene sequencing, ${ }^{4,15-18,24}$ yet $16 \mathrm{~S}$-based classifications cannot discriminate among strains, or even species, within the same bacterial genera, which may substantially differ in terms of immunogenicity. ${ }^{25,26}$ Indeed, Geva-Zatorsky et al. found that two related species of Bacteroides (B. thetaiotaomicron and $B$. vulgatus) displayed differential $\lg A$ induction potential in monocolonized gnotobiotic mice. ${ }^{19}$ By contrast, whole genome shotgun sequencing has extended taxonomic classification at both species and strain level, ${ }^{27}$ thereby revealing $\operatorname{lgA}$ predilections at lower taxonomic levels. In addition, whereas some authors focused their analysis on the $\lg \mathrm{A}^{\text {high }}$ population, ${ }^{3,4,24}$ other studies included both $\lg \mathrm{A}^{\text {high }}$ and $\lg \mathrm{A}^{\operatorname{dim}}$ populations ${ }^{9,17}$ in the definition of $\lg \mathrm{A}^{+}$ microbiota. Beyond these practical issues, interstudy variations also reflect the considerable diversity of human gut microbiota. ${ }^{28,29}$ Numerous factors such as age, diet, antibiotics, and genetic background impact gut microbiota composition. ${ }^{30-33}$ Interestingly, environmental factors have also been shown to influence IgA responses to bacteria. For instance, antibiotic exposure is associated with increased IgA coating of Enterococcus $s p$ and Lactobacillales ${ }^{34}$ and it is therefore expected that each individual harbors a distinct IgA-coated bacterial repertoire. Another potential challenge for IgAseq studies is that enchained growth, driven by high-affinity $\lg \mathrm{A}$, can exert a negative selective pressure. $^{35}$ Enchained growth can also contribute to an environment with lower levels of temperate phage reactivation. ${ }^{36}$ It is therefore expected that bacteria persisting in the gut in the presence of IgA have evolved traits to avoid, evade or benefit from enchained growth (such as large capsules preventing cross-linking and protect from phage predation, or enchained growth-induced microcolony deep mucus settling ${ }^{36,37}$ ). Strains that are susceptible to IgA-mediated enchained growth are expected to be absent from IgAseq data, and are logically extremely difficult to identify by lgAseq alone. We also need to take care in interpreting species presence/absence data in terms of function: the microbiota is a complex ecosystem and minor shifts in the abundance or function of one species can have chaotic effects on the rest of the consortium.

\section{ANALYSIS OF IGA-COATED BACTERIAL COMMUNITIES TO UNRAVEL IGA FUNCTION}

The identification of IgA-coated bacteria has provided new insights in our understanding of the role of secretory $\lg A$ in mucosal homeostasis. The contribution of human studies to describe functions of IgA will be discussed below, while other important roles of secretory $\lg A$ are reviewed elsewhere in this issue.

The work published by Palm et al. suggests that IgA could coat a group of disease-prone intestinal bacteria in humans. ${ }^{24}$ This conclusion is based on human $\lg \mathrm{A}+$ microbiota transfer to murine 
disease models. The latter view proposing that $\lg \mathrm{A}$ would not induce bacterial clearance, but instead might minimize inflammatory effects of targeted bacteria, has been confirmed in other studies in mice. In B. thetaiotaomicron monocolonized mice, Peterson et al. showed that specific lgA selects against a specific phase-variable capsular polysaccharide associated with reduced intestinal innate immune activation. ${ }^{38}$ Affinity-maturated IgA appear also necessary for SFB (Segmented Filamentous Bacteria) pathobiont control, since AID- and IgA-deficient mice suffer from SFB overgrowth and intestinal inflammation. ${ }^{39,40}$ Thus, the selective and/or neutralizing effects of $\lg A$ could modulate bacterial fitness toward a noninflammatory profile. Another interpretation is that bacteria with a better grafting potential in mice have a more pronounced impact on the immune system and therefore are more likely to induce a strong IgA response. These two effects likely coexist in vivo.

These observations notwithstanding, it is also possible that $\lg \mathrm{A}$ might be beneficial for commensal growth and therefore could play a protective role in commensal ecology. In line with this idea, we observed that bacterial species known to be IgA-coated in human samples are more likely to be underrepresented than overrepresented in fecal microbiota from slgAd patients. ${ }^{3}$ Therefore, IgA do not necessarily inhibit bacterial growth, since bacteria such as Coprococcus comes, Dorea sp., and Clostridium sp. thrive in the presence of $\operatorname{lgA} .35$ This promicrobial effect of $\operatorname{lgA}$ has also been shown using an in vitro system, in which human $\lg A$ facilitate biofilm formation and enteric bacteria proliferation. ${ }^{41}$ Interestingly, Donaldson et al. demonstrated that IgA binding to Bacteroides fragilis facilitated adherence to intestinal epithelial cells by modulating surface epitopes ${ }^{37}$ promoting long-term maintenance of this species in the gut microbiota. Furthermore, Nakajima et al. showed that $\lg A$ interacts with $B$. thetaiotaomicron via the $\mathrm{O}$ - and $\mathrm{N}$-glycans that extensively decorate its secretory component and hinge regions. ${ }^{42}$ The latter results also raise the important issue of the relative importance of Fab- (i.e., canonical binding) versus Fc- or glycan-bound (i.e., noncanonical bonding) consortia, which are not currently distinguished in most IgAseq studies.

\section{IGA AS A REGULATOR OF BACTERIAL NETWORKS}

An attractive hypothesis is that $\lg A$ might support bacterial symbiosis by facilitating or modifying bacterial networks. Bacterial symbiosis implies that some bacteria depend on other bacteria for their persistence. Within such interdependence, a bacterium, called "satellite", never inhabits independently of another, named "host", in a given microbiota. In slgAd patients, substantial links were found to be lost between satellites and hosts meaning that bacterial networks are altered in the absence of $\operatorname{lgA} .^{3}$ One could consider the possibility that $\lg \mathrm{A}$, bound noncanonically or displaying cross-species reactivity, might favor incorporation into symbiotic networks as an ecological extension of their ability to agglutinate pathogens. Despite these exciting insights into the role of IgA in microbiota homeostasis, studies in human patients who lack IgA share a main weak spot: such patients were recruited from hospital and therefore may not be representative of the majority of slgAd individuals who are asymptomatic. To better understand the physiological role of $\lg A$ in humans, a major challenge will be to explore a large number of asymptomatic slgAd individuals and to design interventional studies in symptomatic patients. Longitudinal studies would also be relevant to look at the effect of $\lg A$ on strain stability over time.

\section{TOWARD THE DEFINITION OF IGA DEFICIENCIES AT FUNCTIONAL LEVEL}

The impressive development of knowledge surrounding IgAcoated bacterial repertoires may lead to the definition of novel immunopathologic conditions characterized by aberrant patterns of IgA coating. Although such perturbations have already been described in humans, their clinical consequences need to be investigated. Initially, Dzidic et al. reported that low proportions of IgA-coated gut-derived bacteria in infants at 12 months of age preceded the development of allergic asthma. Furthermore, $\lg \mathrm{A}^{+}$repertoires remarkably differed between healthy and allergic children. ${ }^{17}$ Although these results remain correlative, one could speculate that overall reduction of $\lg \mathrm{A}$ coating might reflect weaker stimulation of the mucosal immune system in allergic children. It will be interesting to follow up whether this is due to the presence of less invasive species, or an altered handling by the immune system of very young children at high risk of atopy. Furthermore, fluctuations of lgA+ fraction were measured in multiple sclerosis fecal microbiota depending on disease status (e.g., remission or relapse). The IgA-coated subset tended to increase during remission, whereas significant decrease of IgA-coated bacteria have been found in active patients. Beyond IgA on their own, the authors discovered that IgA-secreting cells travel from the gut to the brain to attenuate neuroinflammation in an interleukin-10 dependent manner. Translating this result from mice to humans, the authors speculated that a reduction in $\lg \mathrm{A}$ bound bacteria might be due to egress of IgA-secreting cells out of the gut. ${ }^{43}$ Aberrant patterns of IgA coating have also been analyzed at the bacterial family level in undernourished children. Gordon et al. reported that Enterobacteriaceae $\operatorname{lgA}$ coating discriminated healthy from undernourished children, suggesting an interrelationship between $\lg \mathrm{A}$ responses and nutritional status. ${ }^{15}$ Further work should investigate whether these changes in $\lg \mathrm{A}$ coating emerge as a consequence of the disease, or play a causative role.

\section{SECRETORY IGM REINFORCES THE ROLE OF SECRETORY IGA}

In addition to IgA-secreting cells, the gut mucosa also contains IgM-secreting cells that coexist with clonally related lgM-memory $B$ cells in humans, but not in mice. ${ }^{4}$ These newly described IgMmemory $B$ cells harbor a gene signature distinct from splenic marginal zone $B$ cells, yet reflecting antigen experience. Both lgMsecreting- and IgM-memory B cells arise as early as 1.5 months after birth. Numbers of IgM-memory B cells remain stable over time, while lgM-secreting cells accumulate over the first 10 years of life. ${ }^{4}$ IgM-secreting cells release slgM into the gut lumen through the polymeric Ig Receptor (plgR). While pentameric IgM shows higher affinity for plgR, export of dimeric $\lg \mathrm{A}$ is favored by a factor 5 . This is likely due to the large size of $\operatorname{lgM}$ limiting its diffusion through the stroma and its access to the plgR. ${ }^{44}$ In contrast to slgA, the extracellular receptor part (secretory component, SC) is noncovalently incorporated to pentameric $\lg M$, which explains why slgM is not as resistant as $\operatorname{sig} A$ to proteolytic degradation in the gut. Importantly, free SC that is released from excess of unoccupied plgR in most secretions, cooperates with bound SC to stabilize the quaternary structure of slgM. $^{45,46}$

Several studies established that slgM play a protective role in gastrointestinal infections. While immune exclusion is mainly performed by slgA, slgM can efficiently bind certain pathogens such as Salmonella enterica Typhimurium and limit their entry into Peyer's patches. ${ }^{47}$ In an experimental Shigella flexneri mouse model, slgM also displayed the ability to modulate the expression of bacterial virulence factors, thereby preserving epithelial surface homeostasis. ${ }^{48}$ Unlike slgA, slgM can activate the complement system, facilitating pathogen killing through the classical pathway and leading to membrane attack complex formation or opsonisation for phagocytosis. Kirkland et al. demonstrated that slgM- and complement-deficiency are associated with a worse disease course of DSS-induced colitis, 
supporting a role for IgM in intestinal homeostasis. However, variations of microbiota in WT versus mutant mice were not studied in these immunodeficient animals. ${ }^{49}$ The protective role of slgM is further illustrated in patients with primary selective IgM deficiency that present increased susceptibility to mucosal infections. ${ }^{50}$

For years, the production of slgM has been considered to function as a compensatory mechanism in patients lacking $\lg \mathrm{A}$, explaining the mild phenotype in the latter. Numerous IgMproducing cells were indeed detected in mucosal samples from slgAd patients compared with healthy donors. ${ }^{1,2}$ Consistently, we found substantial IgM binding to commensals in slgAd fecal samples and higher free fecal IgM levels compared with healthy donors. However, unlike slgA, slgM was found to poorly bind bacteria belonging to Enterobacteriaceae family, which indeed overgrow in slgAd microbiota. These observations could explain the susceptibility to enteropathogens in slgAd patients. ${ }^{3,51} \mathrm{We}$ also observed that slgM binding preserves Actinobacteria diversity, which, strikingly decreased dramatically in patients with common variable immunodeficiency (CVID) patients, lacking both $\lg A$ and $\lg M^{3,52}$ thus highlighting a new role of $\operatorname{sig} M$ in supporting gut microbiota diversity. IgM function is unlikely to be restricted to a particular disease state in humans. Magri et al. indeed profiled mucus-embedded bacteria from ileum and colon of healthy individuals and detected significant IgM-bound commensals. ${ }^{4}$ A greater bacterial diversity has been found in lgM-coated communities, corroborating the notion that slgM optimize microbiota diversity and likely to maximize mucus retention of specific bacterial communities with putative beneficial effects. Accordingly, the microbiota of CVID patients is characterized by a drastic reduction in bacterial diversity and a high dysbiosis index, as compared with slgAd microbiota. ${ }^{52,53}$ Moreover, the prevalence of IBD is higher in CVID patients than in slgAd patients. ${ }^{5}$ Altogether, slgM appear to play a dual role in the intestine, on one hand providing protection from pathogens and on the other hand preserving gut microbiota diversity and intestinal homeostasis, particularly in the case of barrier- or $\lg \mathrm{A}$ insufficiency.

\section{SPATIAL HETEROGENEITY OF SECRETORY IG}

Profiling of Ig-bound commensals enabled by bacterial flow cytometry provides a valuable picture of the spatial heterogeneity of bacterial communities and secretory antibodies abundance. For instance, Ig-bound bacteria are more frequent in ileum than in colon and in mucus compared with central gut lumen. $3,4,9,15,24$ The mucus layer is thin in the small intestine but divided in two distinct layers in colon with an inner, attached layer and an outer, loose mucus layer. Swab samples or colon biopsy were used to profile mucus-associated bacteria, while fecal content served as central gut lumen representative. ${ }^{54-56}$ In the mucus layer, healthy microbiota can be divided into three fractions: $\operatorname{slg} \mathrm{A}^{+} \operatorname{slg} \mathrm{M}^{+}$, $\operatorname{slg} \mathrm{A}^{+} \operatorname{slg} \mathrm{M}^{-}$, and $\operatorname{slg} \mathrm{A}^{-} \operatorname{slg}^{-}$commensals although in central gut lumen slgM coated bacteria have not been detected in healthy donors. Thus, only two fractions can be discriminated: $\operatorname{slg}^{+}$slgM $^{-}$and $\operatorname{slgA}^{-}$slgM $^{-}$bacteria (Fig. 1). ${ }^{3,4}$ One could speculate that slgM cannot be detected in the central gut lumen due to its slow, size-dependent diffusion through mucus and rapid proteolysis, although this remains to be demonstrated in vivo. The existence of an antibody gradient from epithelium to central gut lumen and from proximal to distal compartments could complicate our understanding of secretory Ig function. Secretory lg may operate differently depending on bacterial density, antibody concentration, and an enriched glycoprotein mucus environment. One study revealed that bacterial density determines how slgA clump bacteria. ${ }^{35}$ At high pathogen densities, $\lg \mathrm{A}$ efficiently agglutinates pathogens as they randomly collide in intestinal compartment. However, at lower densities, pathogen collisions are too rare and IgA-mediated clumping can only occur by enchaining growing bacteria (which are necessarily in close proximity ${ }^{35}$ ). Because location will also influence bacterial growth rates, future work should determine how biogeography might have implications for secretory lg functions.

\section{SYSTEMIC IGG RESPONSES TO MICROBIOTA}

The initial observation that patients with IBD presented high titers of serum lgG against intestinal commensal bacteria was thought

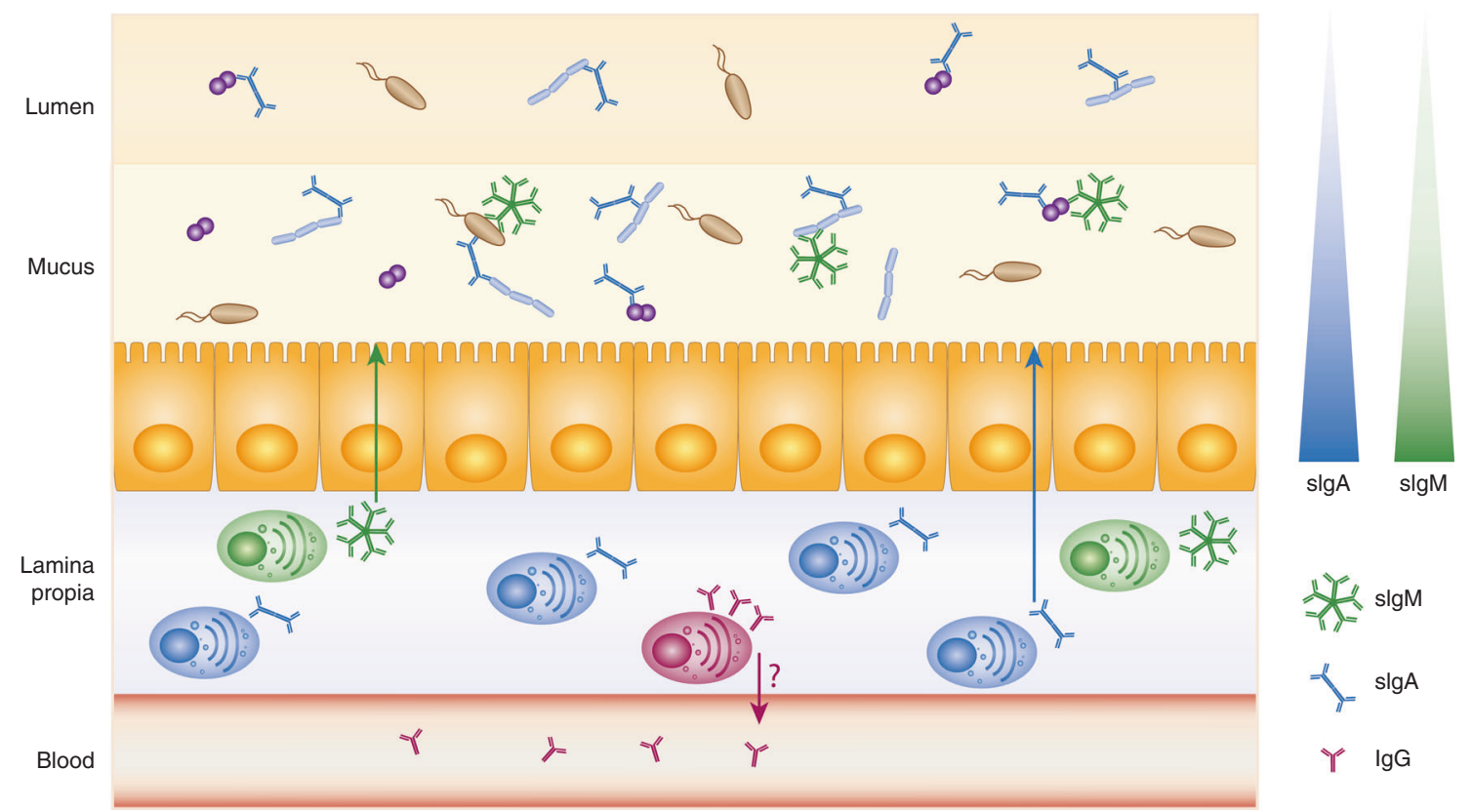

Fig. 1 Secretory and systemic Ig work in concert to control gut microbiota. slgA, slgM, and serum lgG converge toward the same microbial targets. ${ }^{4,62}$ slgA and slgMs are present in decreasing concentrations from mucus to lumen. ${ }^{3,4,9,15,24}$ Although IgG are absent from the healthy human gut, lgG plasmocytes are found in the lamina propria ${ }^{70}$ and likely contribute to systemic anti-commensal lgG pools, whose main origin remains unclear 
to reflect a leaky epithelial barrier in these patients. ${ }^{57,58}$ However, several recent studies have demonstrated that robust levels of serum IgG capable of binding symbiotic bacteria are also detectable in healthy humans. ${ }^{59-62}$ Similarly, peripheral $\mathrm{CD}^{+}$ $\mathrm{T}$ cells specific for intestinal are present in healthy donors. ${ }^{63,64}$ Using novel sensitive assays, such as microbial protein arrays and bacterial flow cytometry, it has been shown that serum lgG levels with measurable affinity for commensals arise by 6 months of age, increase from 12 to 24 months of age and remain stable in adulthood. ${ }^{61,62}$ This timing correlates well with the pattern of gut colonization at birth, diversification of the microbiota after weaning, and microbiota stabilization during adulthood. ${ }^{65,66}$ Longitudinal analysis within a time frame of 5-6 years showed that commensal-binding antibody responses do not significantly change over time in humans. ${ }^{59}$ In line with these data, anticommensal lgG antibodies have been found in naïve mice housed under steady state conditions. ${ }^{12,67}$ In addition, an elegant model of reversible Escherichia coli colonization unequivocally demonstrated that intestinally restrained bacteria are able to induce persistent levels of specific lgG in periphery. ${ }^{68,69}$

Early studies using bacterial flow cytometry showed that systemic IgG bound a wide range of commensal bacteria (E. coli, Enterococcus faecalis, and $B$. fragilis), as well as certain commensal fungi such as Candida albicans. ${ }^{59}$ We recently reported that about $1 \%$ of the gut microbiota of healthy individuals can be bound by serum IgG. Strikingly, serum IgG converge with secretory IgA toward the same bacterial targets with each subject exhibiting a private set of preferred IgG-targeted commensals. However, a "core" IgG repertoire to particular commensals emerged, genera belonging to Proteobacteria (Serratia, Achromobacter and Delftia for instance) being preferentially enriched in the lgG-targeted fraction of the microbiota. ${ }^{62}$ Of note, multiple taxa of the phylum Proteobacteria have been previously described as secretory IgA targets in several human, as well as murine studies. ${ }^{9,14,16}$ Together, these observations suggest that serum IgG coat a particular subset of microbiota, including a broad range of bacteria, enriched in Proteobacteria. Because 16S sequencing analysis cannot resolve species and substrains variations that directly modulate lgG responses, Christmann et al. developed an alternative approach to decipher bacterial determinants of $\mathrm{lgG}$ responses using printing slides with a panel of known antigens from the gut microbiota. ${ }^{61}$ Using this technique, IgG reactivity to different bacterial components, both extracellular and intracellular, from the three most prominent phyla of the human gut microbiota has been uncovered. Four antigens were found to be universally recognized among the cohort. It remains to be shown whether this is due to cross-reactivity with yet unidentified common pathogen antigens, or whether the intriguing hypothesis that postulates the existence of a core microbiota-binding lgG reactivity can be validated. The results of a recent study supported the idea of cross-reactivity of microbiotabinding lgG by showing that monoclonal antibodies specific for the Klebsiella $\mathrm{O} 3 \mathrm{O}$-antigen, a mannose polymer, also bind yeast and mammalian-derived mannose-containing glycans. Such antibodies were shown to bind an array of commensals, likely sharing mannosecontaining surface structures. ${ }^{70}$ The potential role of such specificities in anti-commensal and cross-reactive autoimmunity is extensively reviewed elsewhere.

\section{THE ORIGIN OF SYSTEMIC ANTI-MICROBIOTA IGG}

Several studies explored commensal-binding lgG in mice and humans yet it remains unclear how this systemic response to gut microbiota develops. It likely reflects episodic exposure to gut commensals that breach the intestinal barrier. Increased gut permeability has been observed in human after high-fat meals, alcohol binging or the use of proton pump inhibitors, and nonsteroidal anti-inflammatory drugs absorption. Various intestinal pathogens, including bacteria and viruses, are also able to disrupt the intestinal epithelial barrier. ${ }^{71,72}$ In such conditions, commensal translocation take place in an inflammatory context, likely in the presence of cytokines promoting the production of $\lg$, instead of $\lg A$, in the lamina propria. It is of note that lg gene sequence analysis has revealed that gut lgG- and IgA-secreting cells are not clonally related and therefore originate from independent precursors. ${ }^{73}$ Consistently, direct switching from $\lg \mathrm{M}$ to $\lg \mathrm{A} 1$ or $\lg \mathrm{A} 2$ predominates in colonic mucosa, in contrast to the rare sequential event during which $\lg M$ switches to $\lg A 2$ via $\lg G 2,{ }^{74}$ reinforcing the idea that microbiota-specific $\lg A$ and $\lg G B$ cells arise via independent mechanisms. Experimental data in mouse models have detailed the ontogeny of anti-commensal IgG $B$ cells showing that many commensal-binding antibodies are generated in a T-independent manner dependent on TLR signaling in B cells. These B cells might derive from B1 cells in mice, although the relevance of this finding for human biology remains unclear. ${ }^{12,75}$ The microbiota also nonspecifically supports systemic antibody production through the production of shortchain fatty acids (SCFAs), the major microbial metabolites of dietary fiber fermentation. SCFAs promote B cell differentiation into antibody-secreting cells and boost $B$ cell metabolism resulting in antibody production. 76

Gut-associated lymphoid tissue (GALT) is the primary site of $\lg A$ induction. ${ }^{77}$ By contrast, the extent to which GALT contributes to the IgG repertoire remains still to be determined. Although the intestinal B cell pool is highly dominated by lgA-producing cells, $3-4 \%$ of intestinal antibody-secreting cells express IgG at steady state in humans. About two thirds of monoclonal antibodies derived from intestinal $\mathrm{gGG}^{+}$plasmablasts react with common gut bacteria $^{71-73}$. Moreover, the majority of anti-commensal IgG antibodies were of the lgG2 subclass and lgG2-secreting cells have been detected in the lamina propria throughout the intestine. $^{62}$ In addition, higher serum anti-E. coli IgG levels in patients with IBD were associated with a substantial increase of gut IgG+ B cells. ${ }^{73}$ In mice, mesenteric lymph nodes have also been implicated in generating systemic IgG responses to invasive bacteria. $^{78}$

Together, these data suggest that intestinal $\operatorname{lgG}^{+} \mathrm{B}$ cells might contribute to the serum IgG repertoire in humans (Fig. 1). Recently, Wilmore et al. demonstrated that members of the gut microbiota enhance serum IgA levels and induce large numbers of lgA-secreting cells in the bone marrow. ${ }^{14}$ However, the existence of such a gut/bone marrow axis supporting the production of anticommensal lgG remains to be proved both in humans and in mice.

\section{A RAISON D'ÊTRE FOR ANTI-COMMENSAL IGG}

The functional relevance of systemic anti-commensal lgG has been first revealed by producing evidence for their protective role in gastrointestinal and systemic infections. Mice lacking the innate immune signaling components MyD88 and TRIF spontaneously produce very high levels of microbiota-targeting lgG. Gnotobiotic colonization demonstrated that these responses were $\mathrm{T}$ celldependent and, at least partly, capable of providing protection from sepsis. ${ }^{67,79}$ The clinical relevance of anti-microbiota IgG is consistent with observations that patients with CVID, lacking circulating lgG, but not intestinal $\lg A$, nevertheless suffer from gastrointestinal infections and lymphoproliferative disorders. ${ }^{6,80}$

Anti-commensal IgG also play a role in early life in modulating the development of the neonatal immune system, with both stimulatory and regulatory effects. Maternal lgG is transported to the neonate across the placenta via the neonatal Fc receptor ${ }^{81}$ that also mediates neonatal uptake of maternal milk-ingested IgG into the small intestine. ${ }^{82,83}$ Transfer of lgG during gestation drives the development of intestinal NKp46 ${ }^{+}$innate lymphoid cells (ILC3) and causes changes in the intestinal gene expression profile, in particular genes involved in mucus secretion and metabolism of sugar or dietary xenobiotics whose expression is upregulated in an 
antibody-dependent manner. Moreover, the study of Gomez de Agüero et al. indicated that microbial products from the maternal microbiota, likely bound by $\lg \mathrm{G}$, were transferred to the neonate before birth. Thus, IgG appear to prepare the neonate in utero to the massive bacterial colonization occurring after birth. ${ }^{68}$ The specificity of these IgG transferred to the neonate remains to be defined, but it is tempting to speculate that anti-commensal lgG is an important component.

Anti-commensal lgG ingested from maternal milk, have also been found to have regulatory effects on the neonatal immune system. Maternal milk contains both secretory $\lg A$ and $\lg G$ that cooperate to discourage bacterial translocation and thereby limiting mucosal $\mathrm{T}$ cell responses. In mice lacking maternal antibodies, an increased frequency of T follicular helper cells and germinal center B cells has been described, resulting in an increased antibody production aimed to restore homeostasis. The absence of either IgA or IgG during suckling is sufficient to fuel aberrant immune responses in gut mucosa. ${ }^{12}$

In summary, systemic anti-commensal lgG (i) provide a preexisting set of antibodies against a variety of pathogens and (ii) might play a critical role in modulating immune system development in early life. Although these findings raise exciting possibilities in terms of novel therapeutic strategies, human data are still lacking. At present, multiple clinical trials have failed to demonstrate consistent beneficial effects of pooled human IgG (IVIGs) in sepsis, suggesting that IVIGs do not contain the appropriate specificities. ${ }^{84}$

\section{ANTI-COMMENSAL IGG AND CANCER}

Current evidence supports a model in which some (potentially invasive) commensals are able to induce lgG responses in the context of a healthy microbiota and an intact intestinal barrier. Other microbes however, might only induce systemic responses in the context of dysbiosis or intestinal barrier disorders. This hypothesis has been extensively studied in IBD patients who presented elevated and biased serum anti-commensal lgG responses. ${ }^{57,58,85,86}$ More recently, we reported that slgAd patients have a higher level of anti-commensal lgG, suggesting that bacterial translocation may occur more frequently in the absence of secretory lgA. Moreover, slgAd patients (SlgAd) harbor a different set of anti-commensal lgG, as compared with healthy donors, which is consistent with the presence of a mild dysbiosis. ${ }^{3}$ Greater alterations of gut microbiota composition and diversity have been described in fecal samples from CVID patients, lacking both $\operatorname{lgA}$ and $\operatorname{lgG} .^{52}$ In line with these findings, pooled IgG from healthy donors (IVIGs) poorly bound to fecal bacteria from CVID patients, meaning that these antibodies do not contain appropriate specificities of anti-commensal lgG for the treatment of dysbiotic patients. ${ }^{62}$
In colorectal cancer (CRC), changes in the intestinal tissue result in a leaky gut, with potential aberrant bacterial excursions. ${ }^{87}$ It has also been reported that colorectal CRC is associated with increased prevalence of Fusobacterium nucleatum, a bacteria species with oncogenic potential. ${ }^{88-90}$ A prospective study recently associated a commensal candidate, Streptococcus gallolyticus subspecies gallolyticus (SGG) with the risk to develop CRC, since anti-SGG IgG were more frequently present in individuals who developed cancer later in life (Table 2). ${ }^{91}$

Although a causative link between the presence of such species and CRC has not been demonstrated, certain anti-commensal $\lg A^{92}$ or $\lg G^{91}$ reactivities might constitute potential diagnostic biomarkers for CRC. The detection of anti-commensal lgG might also offer novel opportunities for predicting immunotherapy efficacy in the treatment of cancers. The dominance of distinct commensal species in gut microbiota such as Akkermansia muciniphila and Enterococcus hirae, has been shown to drive immunological changes that influence the anti-cancer efficacy of blockade of the PD1/PDL1 pathway in epithelial tumors. ${ }^{93}$ Screening lgG against these specific microbes before treatment could be more convenient than an extensive analysis of stool composition in an attempt to predict resistance to these and other immune checkpoint inhibitors.

\section{ANTI-COMMENSAL IGG AND AUTOIMMUNITY}

For years, the molecular mimicry theory has supported the view that homologies between microbial components and human proteins are likely to take part in the development of autoimmune diseases. This dogma was first supported by studies showing associations between pathogens and the risk to develop autoimmunity, such as Campylobacter jejuni and Guillain-Barré syndrome or Streptococcus pyogenes and juvenile dermatomyositis. ${ }^{94-96}$ A few recently published studies also aimed at linking the presence of commensals and anti-commensal lgG with the development of certain autoimmune diseases. Recently, Azzouz et al. found that active SLE patients displayed a fecal expansion of Ruminococcus gnavus strain associated with high and specific systemic IgG responses toward this species, suggesting that some specific components of $R$. gnavus are nephritogenic by molecular mimicry (Table 2). ${ }^{97}$ The magnitude of serum antibodies titers directed against Enterococcus gallinarum was correlated with that of anti-RNA antibodies in serum from patients with systemic lupus erythematosus. ${ }^{98}$ Greiling et al. also proposed that commensal bacteria expressing Ro60 orthologs might induce hRo60 cross-reactive antibodies and therefore trigger autoimmunity in lupus. ${ }^{99}$ To test this hypothesis, the authors monocolonized lupus-prone mice with Ro60-expressing bacteria i.e., Bacteroides thetaiotaomicron and observed increased

Table 2. Anti-commensal lgG responses are altered in various diseases

\begin{tabular}{llll}
\hline Disease & $\begin{array}{l}\text { Whole anti-commensal } \\
\text { lgG response }\end{array}$ & Commensal candidates & \\
\hline Selective IgA deficiency & $\nearrow$ & ND & 62 \\
Colorectal cancer & ND & Streptococcus gallolyticus subspecies gallolyticus (SGG) & 87 \\
Systemic lupus erythematosus & ND & $\begin{array}{l}\text { Enterococcus gallinarum } \\
\text { Bacteroides thetaiotaomicron }\end{array}$ & Ruminococcus gnavus \\
Inflammatory bowel diseases & $\nearrow$ & E. coli & \\
& & E. coli, B. fragilis, C. perfringens & \\
& & E. coli & \\
Allergy & CBir1 flagellin & \\
\hline
\end{tabular}

ND not determined 
autoantibody production and lupus-like nephritis. However, most microbes can induce strong immune responses in monocolonized gnotobiotic mice, ${ }^{19}$ likely representing an effect of monocolonization due to overabundance of a single species, or immaturity of the germ-free mucosal system on initial encounter with the microbe. Moreover, autoimmune prone nonobesediabetic mice that were monocolonized with $B$. thetaiotaomicron actually carried hRo60 cross-reactive antibodies but did not develop autoimmune symptoms, indicating that triggering autoimmunity might be the consequence of a much more complex interplay between commensals, host genetics and environmental factors.

Another paradigm, that links anti-commensal lgG responses and immune-mediated diseases is represented by the so-called hygiene hypothesis. ${ }^{100-102}$ Christmann et al. associated lower anti-commensal IgG levels during infancy with the risk to later on eventually develop allergy, suggesting that strong early life anti-microbiota immune responses might be protective against the subsequent development of immune-mediated diseases (Table 2). ${ }^{61}$ Likewise, early colonization by microbes, like Bacteroides sp., that potently activate immune pathways plays an important role in immune education and decreased incidence of autoimmune diseases. ${ }^{103}$ Of note, such induced beneficial immune deviation effects would not be mediated predominantly by pathogens, but rather result from exposure to diverse commensal consortia. ${ }^{104,105}$

\section{OUTLOOK}

The sequencing of immunoglobulin-bound microbiota fractions from both healthy humans and patient populations has provided insight in the nature of bacterial interactions with the host immune system. We and others have noted interesting overlaps and distinctions between $\lg \mathrm{A}, \lg \mathrm{M}$, and $\lg \mathrm{G}$ reactivities, which may hint at both differential induction pathways and differential functions. For example, bacterial strains showing strong reactivity with all three isotypes in healthy donors may be particularly strong inducers of humoral immunity. However, in patients with slgAd there is a counterintuitive (paradoxical) absence of compensatory slgM directed against Gammaproteobacteria, despite evident overgrowth of this gram-negative class of bacteria, suggesting an, as yet, unknown requirement for the induction of slgM. Another challenge remains in interpreting these results with respect to microbiota control. The mechanisms by which $\lg \mathrm{A}$ can selectively disadvantage a bacterial species in the gut lumen are well defined, but while a range of hypotheses exist, there is still limited evidence for procolonization mechanisms. Suggestions currently include the generation of mucus-embedded microcolonies or altered bacterial metabolism. Highly relevant questions also remain regarding the function of anti-commensal Ig in health and disease. Currently, the relatively mild clinical phenotype associated with IgA deficiency is difficult to reconcile with the critical role played by $\lg A$ in disease protection in many animal models. ${ }^{47,106-108}$ Further, microbiota robustness is emerging as an important parameter for health, in which secretory antibodies could play both beneficial and deleterious roles. Perturbation/recovery studies, as well as hypothesis-driven research in experimental model systems, will be required to address these major questions. We would propose these as priorities, necessary to open up the major untapped potential of secretory antibodies in modulating microbiota for diagnostic and therapeutic purposes.

\section{ACKNOWLEDGEMENTS}

We thank members of the Gorochov and Slack labs for discussions and $\mathrm{H}$. Yssel for critical reading of the paper. D.S. was supported for this work by a Pasteur/APHP interface fellowship.

\section{ADDITIONAL INFORMATION}

Competing interests: The authors declare no competing interests.

Publisher's note: Springer Nature remains neutral with regard to jurisdictional claims in published maps and institutional affiliations.

\section{REFERENCES}

1. Brandtzaeg, P. et al. The clinical condition of IgA-deficient patients is related to the proportion of IgD- and IgM-producing cells in their nasal mucosa. Clin. Exp. Immunol. 67, 626-636 (1987).

2. Mellander, L., Björkander, J., Carlsson, B. \& Hanson, L. A. Secretory antibodies in IgA-deficient and immunosuppressed individuals. J. Clin. Immunol. 6, 284-291 (1986).

3. Fadlallah, J. et al. Microbial ecology perturbation in human $\lg \mathrm{A}$ deficiency. Sci. Transl. Med. 10, eaan1217 (2018).

4. Magri, G. et al. Human secretory lgM emerges from plasma cells clonally related to gut memory B cells and targets highly diverse commensals. Immunity 47, 118-134.e8 (2017)

5. Agarwal, S. \& Mayer, L. Pathogenesis and treatment of gastrointestinal disease in antibody deficiency syndromes. J. Allergy Clin. Immunol. 124, 658-664 (2009).

6. Agarwal, S. \& Mayer, L. Diagnosis and treatment of gastrointestinal disorders in patients with primary immunodeficiency. Clin. Gastroenterol. Hepatol. 11, 1050-1063 (2013).

7. Tsuruta, T. et al. The amount of secreted IgA may not determine the secretory IgA coating ratio of gastrointestinal bacteria. FEMS Immunol. Med. Microbiol 56, 185-189 (2009).

8. Waaij, L. A., van der, Limburg, P. C., Mesander, G. \& Waaij, Dvander In vivo IgA coating of anaerobic bacteria in human faeces. Gut 38, 348-354 (1996).

9. Bunker, J. J. et al. Innate and adaptive humoral responses coat distinct commensal bacteria with immunoglobulin A. Immunity 43, 541-553 (2015).

10. Bunker, J. J. et al. Natural polyreactive $\lg A$ antibodies coat the intestinal microbiota. Science. https://doi.org/10.1126/science.aan6619 (2017).

11. Kawamoto, S. et al. Foxp3(+) T cells regulate immunoglobulin a selection and facilitate diversification of bacterial species responsible for immune homeostasis. Immunity 41, 152-165 (2014).

12. Koch, M. A. et al. Maternal lgG and $\lg A$ antibodies dampen mucosal T helper cell responses in early Life. Cell 165, 827-841 (2016).

13. Kubinak, J. L. et al. MHC variation sculpts individualized microbial communities that control susceptibility to enteric infection. Nat. Commun. 6, 8642 (2015).

14. Wilmore, J. R. et al. Commensal microbes induce serum IgA responses that protect against Polymicrobial Sepsis. Cell Host Microbe 23, 302-311.e3 (2018).

15. Kau, A. L. et al. Functional characterization of IgA-targeted bacterial taxa from undernourished Malawian children that produce diet-dependent enteropathy. Sci. Transl. Med. 7, 276 ra24 (2015).

16. Planer, J. D. et al. Development of the gut microbiota and mucosal IgA responses in twins and gnotobiotic mice. Nature 534, 263-266 (2016).

17. Dzidic, M. et al. Aberrant IgA responses to the gut microbiota during infancy precede asthma and allergy development. J. Allergy Clin. Immunol. 139, 1017-1025.e14 (2017).

18. D'Auria, G. et al. Active and secreted IgA-coated bacterial fractions from the human gut reveal an under-represented microbiota core. Sci. Rep. 3, 3515 (2013).

19. Geva-Zatorsky, N. et al. Mining the human gut microbiota for immunomodulatory organisms. Cell 168, 928-943.e11 (2017).

20. Gaboriau-Routhiau, R. S. The key role of segmented filamentous bacteria in the coordinated maturation of gut helper T cell responses. Immunity 31, 677-689 (2009).

21. $\mathrm{Wu}, \mathrm{W}$. et al. Commensal A4 bacteria inhibit intestinal Th2-cell responses through induction of dendritic cell TGF- $\beta$ production. Eur. J. Immunol. 46, 1162-1167 (2016)

22. Liu, X. et al. Role of the gut microbiome in modulating arthritis progression in mice. Sci. Rep. 6, 30594 (2016).

23. Lodes, M. J. et al. Bacterial flagellin is a dominant antigen in Crohn disease. J. Clin. Investig. 113, 1296-1306 (2004).

24. Palm, N. W. et al. Immunoglobulin A coating identifies colitogenic bacteria in inflammatory bowel disease. Cell 158, 1000-1010 (2014).

25. Group, J. C. H. M. P. D. G. W. Evaluation of $16 \mathrm{~S}$ rDNA-based community profiling for human microbiome research. PLos One 7, e39315 (2012).

26. Greenblum, S., Carr, R. \& Borenstein, E. Extensive strain-level copy-number variation across human gut microbiome species. Cell 160, 583-594 (2015).

27. Jovel, J. et al. Characterization of the gut microbiome using $16 \mathrm{~S}$ or shotgun metagenomics. Front. Microbiol. 7, 459 (2016). https://doi.org/10.3389/ fmicb.2016.00459. 
28. Eckburg, P. B. et al. Diversity of the human intestinal microbial flora. Science $\mathbf{3 0 8 ,}$ 1635-1638 (2005).

29. Lozupone, C. A., Stombaugh, J. I., Gordon, J. I., Jansson, J. K. \& Knight, R. Diversity, stability and resilience of the human gut microbiota. Nature 489, 220-230 (2012).

30. Zhernakova, A. et al. Population-based metagenomics analysis reveals markers for gut microbiome composition and diversity. Science 352, 565-569 (2016).

31. Bonder, M. J. et al. The effect of host genetics on the gut microbiome. Nat. Genet. 48, 1407-1412 (2016).

32. $\mathrm{Wu}, \mathrm{G}$. D. et al. Linking long-term dietary patterns with gut microbial enterotypes. Science 334, 105-108 (2011).

33. Ruiz, V. E. et al. A single early-in-life macrolide course has lasting effects on murine microbial network topology and immunity. Nat. Commun. 8, 518 (2017).

34. Džunková, M. et al. Active and secretory lgA-coated bacterial fractions elucidate dysbiosis in clostridium difficile infection. mSphere 1, e00101-e00116 (2016).

35. Moor, K. et al. High-avidity IgA protects the intestine by enchaining growing bacteria. Nature. https://doi.org/10.1038/nature22058 (2017).

36. Diard, M. et al. Inflammation boosts bacteriophage transfer between Salmonella spp. Science 355, 1211-1215 (2017).

37. Donaldson, G. P. et al. Gut microbiota utilize immunoglobulin A for mucosal colonization. Science 360, 795-800 (2018)

38. Peterson, D. A., McNulty, N. P., Guruge, J. L. \& Gordon, J. I. IgA response to symbiotic bacteria as a mediator of gut homeostasis. Cell Host Microbe 2, 328-339 (2007).

39. Fagarasan, S. et al. Critical roles of activation-induced cytidine deaminase in the homeostasis of gut flora. Science 298, 1424-1427 (2002).

40. Suzuki, K. et al. Aberrant expansion of segmented filamentous bacteria in IgAdeficient gut. Proc. Natl Acad. Sci. USA 101, 1981-1986 (2004).

41. Bollinger, R. R. et al. Human secretory immunoglobulin A may contribute to biofilm formation in the gut. Immunology 109, 580-587 (2003).

42. Nakajima, A. et al. IgA regulates the composition and metabolic function of gut microbiota by promoting symbiosis between bacteria. J. Exp. Med. 215, 2019-2034 (2018).

43. Rojas, O. L. et al. Recirculating intestinal IgA-producing cells regulate neuroinflammation via IL-10. Cell 176, 610-624.e18 (2019).

44. Natvig, I. B., Johansen, F. E., Nordeng, T. W., Haraldsen, G. \& Brandtzaeg, P. Mechanism for enhanced external transfer of dimeric IgA over pentameric IgM: studies of diffusion, binding to the human polymeric Ig receptor, and epithelial transcytosis. J. Immunol. 159, 4330-4340 (1997).

45. Brandtzaeg, P. Secretory IgA: designed for anti-microbial defense. Front. Immunol. 4, 222 (2013).

46. Brandtzaeg, P. Human secretory immunoglobulins. 3. Immunochemical and physicochemical studies of secretory lgA and free secretory piece. Acta Pathol. Microbiol. Scand. B Microbiol. Immunol. 79, 165-188 (1971).

47. Bioley, G. et al. Plasma-derived polyreactive secretory-like IgA and IgM opsonizing salmonella enterica typhimurium reduces invasion and gut tissue inflammation through agglutination. Front. Immunol. 8, 1043 (2017).

48. Longet, S. et al. Reconstituted human polyclonal plasma-derived secretory-like $\lg \mathrm{M}$ and $\lg \mathrm{A}$ maintain the barrier function of epithelial cells infected with an enteropathogen. J. Biol. Chem. 289, 21617-21626 (2014).

49. Kirkland, D. et al. B cell-intrinsic MyD88 signaling prevents the lethal dissemination of commensal bacteria during colonic damage. Immunity 36, 228-238 (2012)

50. Chovancova, Z. et al. Selective IgM Deficiency: clinical and laboratory features of 17 patients and a review of the literature. J. Clin. Immunol. https://doi.org/ 10.1007/s10875-017-0420-8 (2017).

51. Elinav, E. et al. NLRP6 inflammasome is a regulator of colonic microbial ecology and risk for colitis. Cell 145, 745-757 (2011).

52. Jørgensen, S. F. et al. Altered gut microbiota profile in common variable immunodeficiency associates with levels of lipopolysaccharide and markers of systemic immune activation. Mucosal Immunol. 9, 1455-1465 (2016).

53. Jørgensen, S. F. et al. Selective IgA deficiency in humans is associated with reduced gut microbial diversity. J. Allergy Clin. Immunol. https://doi.org/10.1016/ j.jaci.2019.01.019 (2019).

54. Bajaj, J. S. et al. Linkage of gut microbiome with cognition in hepatic encephalopathy. Am. J. Physiol. Gastrointest. Liver Physiol. 302, G168-G175 (2012).

55. Richard, M. L. et al. Mucosa-associated microbiota dysbiosis in colitis associated cancer. Gut Microbes .https://doi.org/10.1080/19490976.2017.1379637 (2017).

56. Johansson, M. E. V., Sjövall, H. \& Hansson, G. C. The gastrointestinal mucus system in health and disease. Nat. Rev. Gastroenterol. Hepatol. 10, 352 (2013).

57. Landers, C. J. et al. Selected loss of tolerance evidenced by Crohn's diseaseassociated immune responses to auto- and microbial antigens. Gastroenterology 123, 689-699 (2002)

58. Macpherson, A., Khoo, U. Y., Forgacs, I., Philpott-Howard, J. \& Bjarnason, I. Mucosal antibodies in inflammatory bowel disease are directed against intestinal bacteria. Gut 38, 365-375 (1996).
59. Haas, A. et al. Systemic antibody responses to gut commensal bacteria during chronic HIV-1 infection. Gut 60, 1506-1519 (2011).

60. Harmsen, H. J. M., Pouwels, S. D., Funke, A., Bos, N. A. \& Dijkstra, G. Crohn's disease patients have more lgG-binding fecal bacteria than controls. Clin. Vaccin. Immunol. 19, 515-521 (2012).

61. Christmann, B. S. et al. Human seroreactivity to gut microbiota antigens. J. Allergy Clin. Immunol. 136, 1378-1386.e1-5 (2015).

62. Fadlallah, J. et al. Synergistic convergence of microbiota-specific systemic lgG and secretory IgA. J. Allergy Clin. Immunol. 143, 1575-1585.e4 (2019).

63. Bradley, C. P. et al. Segmented filamentous bacteria provoke lung autoimmunity by inducing gut-lung Axis Th17 cells expressing dual TCRs. Cell Host Microbe 22, 697 (2017).

64. Hegazy, A. N. et al. Circulating and tissue-resident CD4+ T cells with reactivity to intestinal microbiota are abundant in healthy individuals and function is altered during inflammation. Gastroenterology 153, 1320-1337.e16 (2017).

65. Lim, E. S. et al. Early life dynamics of the human gut virome and bacterial microbiome in infants. Nat. Med. 21, 1228-1234 (2015).

66. Faith, J. J. et al. The long-term stability of the human gut microbiota. Science 341, 1237439 (2013)

67. Zeng, M. Y. et al. Gut microbiota-induced immunoglobulin $\mathrm{G}$ controls systemic infection by symbiotic bacteria and pathogens. Immunity 44, 647-658 (2016).

68. Gomez de Agüero, M. et al. The maternal microbiota drives early postnatal innate immune development. Science 351, 1296-1302 (2016).

69. Hapfelmeier, S. et al. Reversible microbial colonization of germ-free mice reveals the dynamics of IgA immune responses. Science 328, 1705-1709 (2010).

70. Rollenske, T. et al. Cross-specificity of protective human antibodies against Klebsiella pneumoniae LPS O-antigen. Nat. Immunol. 19, 617-624 (2018).

71. Bala, S., Marcos, M., Gattu, A., Catalano, D. \& Szabo, G. Acute binge drinking increases serum endotoxin and bacterial DNA levels in healthy individuals. PLOS One 9, e96864 (2014).

72. König, J. et al. Human intestinal barrier function in health and disease Clinical and Translational. Gastroenterol 7, e196 (2016).

73. Benckert, J. et al. The majority of intestinal $\lg \mathrm{A}+$ and $\lg \mathrm{G}+$ plasmablasts in the human gut are antigen-specific. J. Clin. Investig. 121, 1946-1955 (2011).

74. Lin, M., Du, L., Brandtzaeg, P. \& Pan-Hammarström, Q. IgA subclass switch recombination in human mucosal and systemic immune compartments. Mucosal Immunol. 7, 511-520 (2014).

75. Descatoire, M., Weill, J.-C., Reynaud, C.-A. \& Weller, S. A human equivalent of mouse B-1 cells? J. Exp. Med. 208, 2563-2564 (2011).

76. Kim, M., Qie, Y., Park, J. \& Kim, C. H. Gut microbial metabolites fuel host antibody responses. Cell Host Microbe 20, 202-214 (2016).

77. Lécuyer, E. et al. Segmented filamentous bacterium uses secondary and tertiary lymphoid tissues to induce gut $\operatorname{lgA}$ and specific $\mathrm{T}$ helper 17 cell responses. Immunity 40, 608-620 (2014).

78. Martinoli, C. et al. Entry route of salmonella typhimurium directs the type of induced immune response. Immunity 27, 975-984 (2007).

79. Slack, E. et al. Innate and adaptive immunity cooperate flexibly to maintain hostmicrobiota mutualism. Science 325, 617-620 (2009).

80. Oksenhendler, E. et al. Infections in 252 patients with common variable immunodeficiency. Clin. Infect. Dis. 46, 1547-1554 (2008).

81. Leach, J. L. et al. Isolation from human placenta of the $\lg G$ transporter, FcRn, and localization to the syncytiotrophoblast: implications for maternal-fetal antibody transport. J. Immunol. 157, 3317-3322 (1996).

82. Roopenian, D. C. \& Akilesh, S. FcRn: the neonatal Fc receptor comes of age. Nat. Rev. Immunol. 7, 715-725 (2007).

83. Pyzik, M., Rath, T., Lencer, W. I., Baker, K. \& Blumberg, R. S. FcRn: the architect behind the immune and nonimmune functions of IgG and albumin. J. Immunol. 194, 4595-4603 (2015).

84. Almansa, R. et al. The original sins of clinical trials with intravenous immunoglobulins in sepsis. Crit. Care 19, 90 (2015).

85. Beaugerie, L. \& Sokol, H. Clinical, serological and genetic predictors of inflammatory bowel disease course. World J. Gastroenterol. 18, 3806-3813 (2012).

86. Targan, S. R. et al. Antibodies to CBir1 flagellin define a unique response that is associated independently with complicated Crohn's disease. Gastroenterology 128, 2020-2028 (2005).

87. Brenner, H., Kloor, M. \& Pox, C. P. Colorectal cancer. Lancet 383, 1490-1502 (2014).

88. Rubinstein, M. R. et al. Fusobacterium nucleatum promotes colorectal carcinogenesis by modulating $\mathrm{E}$-cadherin/ $\beta$-catenin signaling via its FadA adhesin. Cell Host Microbe 14, 195-206 (2013).

89. Kostic, A. D. et al. Fusobacterium nucleatum potentiates intestinal tumorigenesis and modulates the tumor-immune microenvironment. Cell Host Microbe 14, 207-215 (2013).

90. Brennan, C. A. \& Garrett, W. S. Fusobacterium nucleatum-symbiont, opportunist and oncobacterium. Nat. Rev. Microbiol. 17, 156-166 (2019). 
91. Butt, J. et al. Prospective evaluation of antibody response to Streptococcus gallolyticus and risk of colorectal cancer. Int. J. Cancer 143, 245-252 (2018).

92. Wang, H.-F. et al. Evaluation of antibody level against Fusobacterium nucleatum in the serological diagnosis of colorectal cancer. Sci. Rep. 6, 33440 (2016).

93. Routy, B. et al. Gut microbiome influences efficacy of PD-1-based immunotherapy against epithelial tumors. Science 359, 91-97 (2018).

94. Cusick, M. F., Libbey, J. E. \& Fujinami, R. S. Molecular mimicry as a mechanism of autoimmune disease. Clin. Rev. Allergy Immunol. 42, 102-111 (2012).

95. Massa, M. et al. Self epitopes shared between human skeletal myosin and streptococcus pyogenes M5 protein are targets of immune responses in active juvenile dermatomyositis. Arthritis Rheumatol. 46, 3015-3025 (2002).

96. Jacobs, B. C. et al. Campylobacter jejuni infections and anti-GM1 antibodies in Guillain-Barré syndrome. Ann. Neurol. 40, 181-187 (1996).

97. Azzouz, D. et al. Lupus nephritis is linked to disease-activity associated expansions and immunity to a gut commensal. Ann. Rheum. Dis.https://doi.org/ 10.1136/annrheumdis-2018-214856 (2019).

98. Manfredo Vieira, S. et al. Translocation of a gut pathobiont drives autoimmunity in mice and humans. Science 359, 1156-1161 (2018).

99. Greiling, T. M. et al. Commensal orthologs of the human autoantigen Ro60 as triggers of autoimmunity in lupus. Sci. Transl. Med. 10, eaan2306 (2018).

100. Bach, J.-F. The hygiene hypothesis in autoimmunity: the role of pathogens and commensals. Nat. Rev. Immunol. 18, 105-120 (2018).
101. Bach, J.-F. The effect of infections on susceptibility to autoimmune and allergic diseases. N. Engl. J. Med. 347, 911-920 (2002).

102. Strachan, D. P. Hay fever, hygiene, and household size. BMJ 299, 1259-1260 (1989).

103. Vatanen, T. et al. Variation in microbiome LPS immunogenicity contributes to autoimmunity in humans. Cell 165, 1551 (2016).

104. Scudellari, M. News Feature: cleaning up the hygiene hypothesis. Proc. Natl Acad. Sci. USA 114, 1433-1436 (2017).

105. Bloomfield, S. F. et al. Time to abandon the hygiene hypothesis: new perspectives on allergic disease, the human microbiome, infectious disease prevention and the role of targeted hygiene. Perspect. Public Health 136, 213-224 (2016).

106. Boullier, S. et al. Secretory IgA-mediated neutralization of Shigella flexneri prevents intestinal tissue destruction by down-regulating inflammatory circuits. $J$. Immunol. 183, 5879-5885 (2009).

107. Winner, L. et al. New model for analysis of mucosal immunity: intestinal secretion of specific monoclonal immunoglobulin A from hybridoma tumors protects against Vibrio cholerae infection. Infect. Immun. 59, 977-982 (1991).

108. Forbes, S. J., Eschmann, M. \& Mantis, N. J. Inhibition of Salmonella enterica serovar typhimurium motility and entry into epithelial cells by a protective antilipopolysaccharide monoclonal immunoglobulin A antibody. Infect. Immun. 76, 4137-4144 (2008) 